\title{
Estimulación ventricular derecha multisitio en insuficiencia cardíaca refractaria a tratamiento con resincronización biventricular
}

Ismael Vergara S, Patricia Frangini S, Rolando González A.

\author{
Multisite right ventricular stimulation \\ for cardiac failure refractory \\ to biventricular resynchronization \\ therapy. Case report
}

The prevalence of congestive heart failure has increased in the world. Despite advances in pharmacological treatment, some patients have progression of the disease and deterioration of their functional class. In this group of patients cardiac resynchronization therapy has been accepted as a treatment option. However, some patients are non-responders to cardiac resynchronization, and others who respond favorably, will experience reappearance of their symptoms. For these patients, multisite stimulation with the implant of a second electrode in the right ventricle has been published as a new option. We report a 76 year-old woman with a dilated cardiomyopathy, who was treated with resynchronization therapy with good clinical response during two years, but symptoms of congestive heart failure reappeared and her functional class deteriorated to NYHA class IV. She was successfully treated with right ventricular multisite stimulation, with a reduction of symptoms that has lasted during the two months of follow up after the procedure (Rev Méd Chile 2006; 134: 629-34).

(Key words: Cardiomiopathy, dilated; Electric stimulation; Heart failure, congestive)

Recibido el 15 de julio, 2005. Aceptado el 8 de septiembre, 2005.

Laboratorio de Electrofisiología Cardíaca, Departamento de Enfermedades Cardiovasculares, Facultad de Medicina, Pontificia Universidad Católica de Chile. Santiago de Chile.

L

a prevalencia de la insuficiencia cardíaca congestiva ha aumentado significativamente en las últimas décadas. Su mortalidad está ligada al grado de compromiso de la función ventricular izquierda, llegando a ser 30 a $50 \%$ a cinco años,

Correspondencia a: Dr. Ismael Vergara S. Marcoleta $3672^{0}$ piso. Teléfono: 3546402. Fax: 6327306. E mail: ivergara@med.puc.cl en el grupo de mayor gravedad ${ }^{1,2}$. El tratamiento farmacológico de la insuficiencia cardíaca ha incorporado drogas con efecto biológico y clínico aceptado, como los inhibidores de la enzima convertidora, bloqueadores de los receptores de angiotensina, antagonistas de la aldosterona y nuevos betabloqueadores con propiedades vasodilatadoras $^{3}$, sin embargo, algunos pacientes permanecen refractarios o progresan en su enfer- 
medad, requiriendo drogas vasoactivas endovenosas o trasplante cardíaco. En los pacientes con miocardiopatía dilatada e insuficiencia cardíaca con capacidad funcional III a IV (según la clasificación de la New York Heart Association), la terapia de estimulación con resincronización biventricular ha surgido como una alternativa de tratamiento, validada por sus efectos sobre parámetros clínicos y la sobrevida de los pacientes ${ }^{4}$.

Presentamos el caso clínico de una paciente con miocardiopatía dilatada sometida a terapia de resincronización biventricular con buena respuesta clínica, quien en su seguimiento evolucionó con deterioro progresivo de su capacidad funcional, por lo que se le ofreció asociar estimulación multisitio en el ventrículo derecho.

\section{CASO CĹNICO}

Mujer de 76 años, con antecedente de miocardiopatía dilatada idiopática de 10 años de evolución. En 1998 presentó cuadro de rápido deterioro de su capacidad funcional, asociado a episodios de fibrilación auricular paroxística con respuesta ventricular acelerada y bradicardia sintomática secundaria a enfermedad del nódulo sinusal, por lo cual se le implantó marcapaso definitivo bicameral, con electrodos endocavitarios en la orejuela derecha y en el ápex del ventrículo derecho. Se mantuvo en relativas buenas condiciones generales, en capacidad funcional I-II, en tratamiento médico con: carvedilol, losartan, isosorbide, digoxina, furosemida y anticoagulación oral. Su ecocardiograma en 2001 mostró dilatación ventricular izquierda (diámetro sistólico 59 mm y diastólico $68 \mathrm{~mm}$ ) y disfunción ventricular (fracción de acortamiento 13\%). A pesar de optimización de las dosis de sus medicamentos, evolucionó con deterioro de su capacidad funcional, llegando a disnea de mínimos esfuerzos y fibrilación auricular crónica con buen control de la frecuencia. En 2003, se sometió a upgrade a marcapaso de resincronización biventricular (Saint Jude ${ }^{\circledR}$, Frontier 5510). Se intentó implante endocavitario del electrodo de estimulación ventricular izquierdo, el que fue frustro debido a que el seno coronario estaba trombosado; en el mismo procedimiento se implantó nuevo electrodo endocavitario de estimulación en el ápex del ventrículo derecho, porque se confirmó daño del aislamiento del electrodo ventricular antiguo. Durante esa hospitalización se implantó electrodo epicárdico bipolar de estimulación ventricular izquierda (Medtronic ${ }^{\circledR}$ 4968), por minitoracotomía izquierda. La paciente no fue cardiovertida y fue dada de alta en buenas condiciones a las $48 \mathrm{~h}$ post-cirugía. El marcapaso fue programado en modo VVT y la paciente evolucionó con franca mejoría sintomática, recuperando capacidad funcional II. Se mantuvo estable hasta fines de 2004, cuando reapareció deterioro de su capacidad funcional con disnea de mínimos esfuerzos y edema de extremidades inferiores, a pesar de mantener su tratamiento farmacológico en dosis máximas toleradas (furosemida $40 \mathrm{mg}$, isosorbide $10 \mathrm{mg} \mathrm{x} \mathrm{2,} \mathrm{digoxina}$ 0,125 mg, losartan $50 \mathrm{mg} \mathrm{x} \mathrm{2,} \mathrm{carvedilol} \mathrm{12,5} \mathrm{mg} \mathrm{x}$ 2, amiodarona $200 \mathrm{mg}$, anticoagulante oral), y de confirmarse funcionamiento adecuado de su marcapaso de resincronización biventricular. No se detectó factor descompensante intercurrente. La paciente fue hospitalizada para reevaluación, su ecocardiograma mostró dilatación y disfunción ventricular significativa (diámetro de 64/59 mm, fracción de acortamiento de 7\%), insuficiencia mitral y tricuspídea moderada y adecuada sincronía entre la pared lateral del ventrículo izquierdo y el septum. Se desestimó intentar cardioversión eléctrica $y$, antes de intentar infusión de inótropos endovenosos, se le ofreció como alternativa de excepción probar si el implante de un segundo electrodo de estimulación ventricular derecha podría ayudar al alivio de sus síntomas. Con este fin se implantó un tercer electrodo de estimulación bipolar en la región septoinfundibular del ventrículo derecho (Saint Jude ${ }^{\circledR}$ 1688T), mediante punción con técnica de Seldinger de la vena subclavia izquierda (Figura 1). Se conservó el dispositivo de estimulación biventricular Saint Jude ${ }^{\circledR}$ Frontier 5510. El electrodo epicárdico de estimulación ventricular izquierda y el nuevo electrodo de estimulación ventricular septoinfundibular derecho se conectaron a través de un adaptador en $₫ \gg$ (Oscor 2x.Bis/Bis-7) al puerto de conexión para el electrodo ventricular izquierdo, manteniendo la conexión del electrodo apical derecho en su puerto original de conexión. El electrocardiograma pre y post procedimiento se muestra en la Figura 2. La paciente fue dada de alta manteniendo su terapia farmacológica, con mejoría sintomá- 


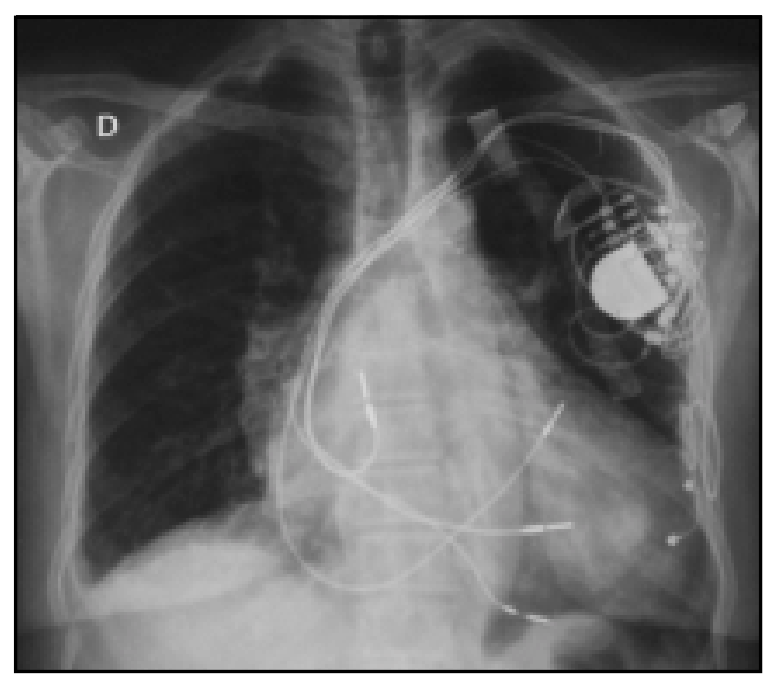

Figura 1. Radiografía de tórax anteroposterior. Se observan 5 electrodos de estimulación cardíaca, 4 endocavitarios y 1 epicárdico. Los electrodos endocavitarios se ubican en: orejuela de la aurícula derecha, región medioseptal del ventrículo derecho (electrodo abandonado), ápex del ventrículo derecho y en el tracto de salida del ventrículo derecho (electrodo implantado para estimulación multisitio). El electrodo epicárdico bipolar se encuentra en la cara lateral del ventrículo izquierdo. Se observa el generador en la región prepectoral izquierda con tres puertos de conexión.

Figura 2. A. Electrocardiograma con estimulación biventricular. B. Electrocardiograma con estimulación biventricular asociada a estimulación multisitio del ventrículo derecho. Se observa un discreto cambio en el vector de estimulación cardíaca, con aparición de $\ll \gtrsim$ en el complejo QRS en V1. Ambos electrocardiogramas fueron registrados durante el implante, sin variación de los electrodos ni de la posición de la paciente, y con la misma estandarización.

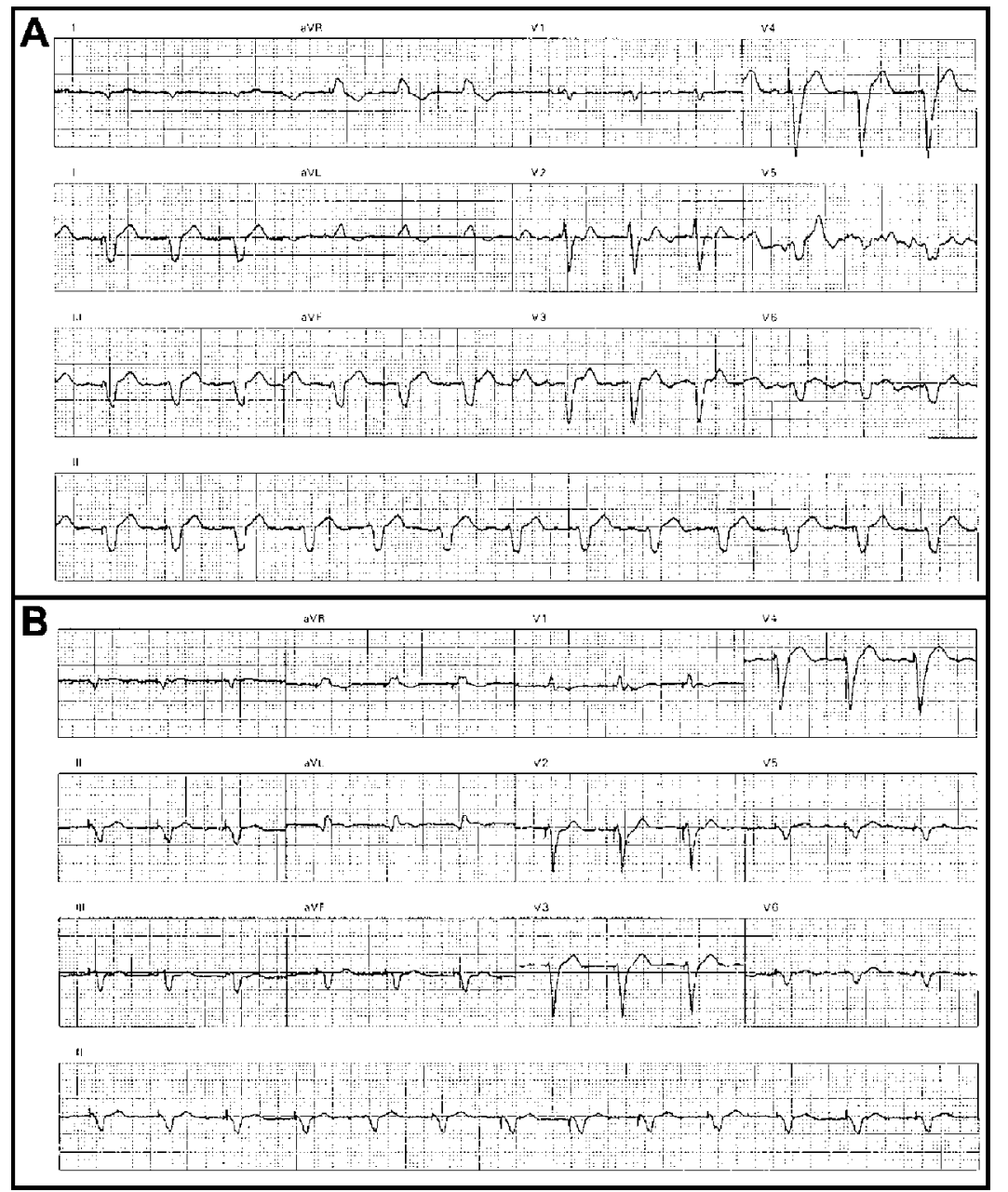


tica, con capacidad funcional II-III, sin reproducir síntomas ni edema a dos meses de seguimiento.

\section{DisCUSIÓN}

Varios estudios han demostrado que los pacientes sometidos a terapia de resincronización biventricular, asociada a terapia farmacológica óptima, presentan una significativa mejoría de sus parámetros clínicos, regresando en al menos una clase funcional de la NYHA y rindiendo mejor en el test de caminata de seis minutos y en la medición de consumo máximo de $\mathrm{O}_{2}$, lo que se asocia a alivio de los síntomas, mejoría en las encuestas de calidad de vida y reducción en el número de hospitalizaciones ${ }^{5-10}$. Los resultados favorables de esta terapia se mantienen a 1 y 2 años plazo ${ }^{11,12}$. La paciente presentada en esta publicación tenía fibrilación auricular de base y fue sometida a terapia de resincronización biventricular, con muy buena respuesta clínica, sin embargo, al año y medio de seguimiento sus síntomas de insuficiencia cardíaca progresaron a capacidad funcional IV, pese a mantener excelente adherencia al tratamiento farmacológico.

Las razones que se han argumentado para explicar el efecto positivo de la resincronización biventricular en pacientes con miocardiopatía dilatada y trastorno de la activación intraventricular, se basan en la corrección del retardo de la contracción intraventricular e interventricular que puede existir en ellos, asociado a mejoría de la sincronía aurículo-ventricular y disminución de la insuficiencia mitral sistólica y también diastólica, lo que se traduciría en aumento en la fracción de eyección del ventrículo izquierdo, remodelamiento cardíaco positivo, aumento de la presión arterial, aumento de la presión de perfusión tisular y disminución de la presión auricular ${ }^{13}$. La respuesta favorable de estos pacientes se puede ver complicada por la evolución natural de la enfermedad, como creemos que ocurrió en nuestra paciente, por la falta de adherencia al tratamiento (que es la principal causa de descompensación en la práctica clínica), por una programación inadecuada, o por la aparición de trastornos que interrumpen el funcionamiento normal del dispositivo. Los parámetros de programación pueden tener un alto grado de influencia sobre la evolu- ción del paciente dado que, por ejemplo, la relación aurículo-ventricular óptima es distinta en presencia de ritmo sinusal y sensado auricular que en presencia de estimulación auricular, ya que el retardo interauricular exagerado por la estimulación artificial puede hacer perder la sincronía $\mathrm{A}-\mathrm{V}$ y el aporte de la contracción auricular izquierda al llenado ventricular izquierdo ${ }^{14,15}$. En un subanálisis del Ventak CHF/Contak CD Biventricular Pacing Study ${ }^{16}$, en un seguimiento a dos y medio años de los 443 pacientes con implante exitoso, $36 \%$ sufrieron alguna interrupción de la estimulación programada debido principalmente a la aparición de arritmias auriculares, especialmente fibrilación auricular (18\%), pérdida de captura ventricular izquierda (10\%), y pérdida de estimulación ventricular derecha (2\%). En la paciente de esta publicación no hubo falla de los umbrales de estimulación ventricular y su fibrilación auricular de base se encontraba con adecuado control de frecuencia, lo que fue varias veces corroborado por los histogramas de su marcapaso que mostraban $100 \%$ de estimulación ventricular. Respecto a las dudas que se han planteado sobre el beneficio de la terapia de resincronización en pacientes con fibrilación auricular de base, la excelente respuesta clínica inicial de la paciente apoya la idea de que ésta también es una terapia útil en este subgrupo de pacientes ${ }^{17}$. Otro elemento a destacar en la historia clínica es el beneficio obtenido al corregir, mediante el implante de un electrodo ventricular izquierdo, la asincronía iatrogénica provocada por la estimulación ventricular derecha exclusiva, lo que concuerda con los resultados favorables de series limitadas de pacientes sometidos a upgrade a un sistema biventricular ${ }^{17,18}$.

El nuevo deterioro en la capacidad funcional, sin otra causa aparente más que la evolución natural de la enfermedad, obligó a buscar alternativas terapéuticas de excepción. En presencia de fibrilación auricular el intervalo AV no es optimizable, y su modelo de marcapaso no permite programación del intervalo interventricular. Hay limitada experiencia clínica reportada sobre la utilidad de asociar estimulación ventricular derecha en dos sitios a un marcapaso de resincronización. Los resultados presentados han sido favorables y así se le planteó a la paciente. En el congreso de la Heart Rhythm Society en mayo de 2005, se presentaron dos resúmenes con esta 
modalidad de estimulación ${ }^{19,20}$, en uno de los cuales se dio cuenta del mejor rendimiento hemodinámico agudo de la estimulación ventricular derecha multisitio sobre la biventricular, asociado a mejoría de la capacidad funcional basal. Otra publicación muestra el efecto positivo de la estimulación multisitio en ventrículo derecho en una serie de pacientes, en los que fracasó el implante endocavitario del electrodo de seno coronario para resincronización ${ }^{21}$. La potencial explicación fisiopatológica para el beneficio de esta modalidad de tratamiento estaría dada por la adición de una mejoría del acortamiento longitudinal del ventrículo (asincronía entre los segmentos basales y apicales), asociada a la mejoría de la

\section{REFERENCIAS}

1. Benatar D, Bondmass M, Ghitelman J, Avitall B. Outcomes of chronic heart failure. Arch Intern Med 2003; 163: 347-52.

2. ANSARI M, Marie BM. Heart failure: How big is the problem? Who are the patients? What does the future hold? Am Heart J 2003; 146: 1-4.

3. Poole-Wilson PA, Swedberg K, Cieiand JGF, Di Lenarda A, Hanrath P, Komajda M et al, for the COMET INVESTIGATORS. Comparison of carvedilol and metoprolol on clinical outcomes in patients with chronic heart failure (COMET): a randomized controlled trial. Lancet 2003; 362: 7-13.

4. Cleland J, Daubert JC, Erdmann E, Freemantie N, Gras D, KappenBerger L et al. The effect of cardiac resynchronization on morbidity and mortality in heart failure. N Engl J Med 2005; 352: 1539-49.

5. Cazeau S, Leclerco C, Lavergne T, Walter S, Varma C, LNNDE C et aL, For MUSTIC Study Investigators. Effects of multisite biventricular pacing in patients with heart failure and intraventricular conduction delay. N Engl J Med 2001; 344: 873-80.

6. Abraham WT, Fisher WG, Smith AL, Delurgio DB, Leon AR, Loh E ET AL, FOR THE MIRACLE Study GROUP. Cardiac resynchronization in chronic heart failure. N Eng J Med 2002; 346: 1845-53.

7. Bradley JB, Bradley EA, Baughman KL, Berger RD, Calkins H, Goodman SN et al. Cardiac resynchro- asincronía intra e interventricular que otorga la resincronización biventricular, como lo demuestran algunas evaluaciones ecocardiográficas con técnicas de Doppler tisular y strain ${ }^{19}$, técnicas que no fueron utilizadas para comparación pre y post procedimiento en el caso que presentamos.

La paciente, a dos meses post-procedimiento, se ha mantenido con su terapia farmacológica sin modificaciones, en capacidad funcional II a III, por lo que pensamos que esto no puede atribuirse simplemente a efecto placebo, y que la estimulación multisitio en ventrículo derecho asociada a resincronización biventricular debería ser evaluada como una alternativa terapéutica útil en pacientes refractarios. nization and death from progressive heart failure. A meta-analysis of randomized controlled studies. JAMA 2003; 289: 730-40.

8. Young JB, Abraham WT, Smith AL, León AR, LEBERMAN R, WiLKOFF B ET AL, FOR THE MIRACLE ICD TRIal InveStigators. Combined cardiac resynchronization and implantable cardioversion defibrillation in advanced chronic heart failure. The MIRACLE ICD trial. JAMA 2003; 289: 2685-94.

9. LECLERCQ C, KASS DA. Retiming the mailing heart: principles and current clinical status of cardiac resynchronization. J Am Coll Cardiol 2002; 39: 194-201.

10. Bristow MR, Saxon LA, Boehmer J, Krueger S, Kass DA, DeMarCO T ET AL, FOR THE COMPANION INVESTIGATORS. Cardiac-resynchronization therapy with or without an implantable defibrillator in advanced chronic heart failure. N Engl J Med 2004; 350: 2140-50.

11. Lunde C, Leclerco C, Rex S, Garrigue S, Lavergne T, Cazeau S et al, (MUSTIC Study Group). Long-term benefits of biventricular pacing in congestive heart failure: Results from the Multisite Stimulation in Cardiomyopathy (MUSTIC) Study. J Am Coll Cardiol 2002; 40: 111-8.

12. Linde C, Braunschweig F, Gadier F, Baileul $C$, DAUBERT JC. Long-term improvement in quality of life by biventricular pacing in patients with chronic heart failure: Results from the MUSTIC study. Am J Cardiol 203; 91: 1090-95. 
13. Yu CM, Chau E, Sanderson JE, Fan K, Tang MO, Fung WH ET AL. Tissue Doppler echocardiographic evidence of reverse remodeling and improved synchronicity by simultaneously delaying regional contraction after biventricular pacing therapy in heart failure. Circulation 2002; 105 : 438-45.

14. Wish M, Fletcher RD, Gottdiener JS, Cohen AI. Importance of left atrial timing in the programming of dual-chamber pacemakers. Am J Cardiol 1987; 60: 566-71.

15. Janosik DL, Pearson AC, Buckingham TA, Labovitz AJ, REDD RM. The hemodynamic benefit of differential atrioventricular delay intervals for sensed and paced atrial events during physiologic pacing. J Am Coll Cardiol 1989; 14: 499-507.

16. Knight BP, Coman J, FAdisM, Yong P. Long-term retention of cardiac resynchronization therapy. J Am Coll Cardiol 2004; 44: 72-7.

17. León AR, Greenberg JM, Kanuru N, Baber CM, Mera FV, SMITH AL ET AL. Cardiac resynchronization in patients with congestive heart failure and chronic atrial fibrillation: Effect of upgrading to biventri- cular pacing after chronic right ventricular pacing. J Am Coll Cardiol 2002; 39: 1258-63.

18. Montagna R, Asenjo $R$, Morris $R$, Schumacher E, Aguayo R, Ortiz M et al. [Ventricular resynchronization therapy in patients with previous pacemaker for the management of advanced refractory heart failure]. Rev Chil Cardiol 2005; 24: 48-59.

19. Yokoyama Y, Aonuma K, TaKahashi A, Kuwahara T, Kobori A, Sato A ET al. Effective cardiac resynchronization with right ventricular outflow tract pacing as the third pacing site additionally to biventricular pacing. Heart Rhythm 2005; 2: S59.

20. Aonuma K, Yokoyama Y, Seo Y, Ohtsuka S, Kawano S, SAтOH A ET AL. Tri-ventricular pacing: a novel concept of resynchronization therapy for better left ventricular performance in end-stage heart failure. Heart Rhythm 2005; 2: S131.

21. O’Donnell D, Nadutara V, Hamer A, Kertes P, MOHAMmed W. Bifocal right ventricular cardiac resynchronization therapies in patients with unsuccessful percutaneous lateral left ventricular venous access. Pacing Clin Electrophysiol 2005; 28: S28-S30. 